\title{
Kinetic modeling of Immobilized Yeast Batch Fermentation
}

\author{
Terkida Prifti (Vaso) $^{1}$, Luljeta Pinguli ${ }^{2}$, Ilirjan Malollari ${ }^{3}$
}

\author{
Department of Industrial Chemistry, Faculty of Natural Sciences, University of Tirana, Tirana, Albania
}

\begin{abstract}
The main purpose of this study was to choose the best model that will be further refined and used for controlling batch fermentation process using immobilised yeast cell in order to increase its productivity. This study investigated the immobilized Saccharomyces Carlsbergensis growth kinetics, ethanol productivity and substrate consumption (glucose) using computer simulation for different kinetic models, Monod, Contois, Teisser and other models which present the substrate and product inhibition. Batch fermentations take place with different cell density comparing immobilized yeast fermentation performance with free yeast. For each batch were evaluated kinetic parameters and after mathematical models. We have used three linearization models for estimation of kinetic parameters. Conclusions about the influence of cell immobilization on the fermentation performance were drawn based on analysis of the obtained model parameters. It was found that in some fermentation Monod and Teisser models were the most appropriate. For substrate and product inhibition the most approximate models were exponential.
\end{abstract}

Keywords — Immobilization, inhibition, kinetic parameters, linearization, modeling.

\section{INTRODUCTION}

The environmental problems caused by the use of fossil feedstock as an energy source and the rapid increase of the oil based fuels prices are the main reasons that have motivated the production of bio-fuels. Bio-ethanol, being a clean, safe and renewable resource, has been considered as a potential alternative to the ever decreasing fossil fuels. Ethanol production has increased dramatically during the last years, because it is considered as a renewable and environmentally friendly alternative. However, the economic feasibility of the ethanol industry is still questioned and much effort should be put into improving the process, especially resistance to the main inhibition factors.

To eliminate inhibition caused by high concentrations of substrate and product as well as to enhance yield, cell immobilization approaches have been applied in ethanol production. The advantages of immobilized cell over free cell systems have been extensively reported. Immobilized cell fermentation has been shown to be more effective than the free yeast process, mainly due to the enhanced fermentation productivity, feasibility for continuous processing, cell stability and lower costs of recovery and recycling and downstream processing. However, immobilized cells still have limited industrial application. The process of immobilization changes not only the environment, but also the physiological and morphological characteristic of cells, and the catalytic activity of enzymes. Therefore the fermentation conditions (kinetics) of the free yeast fermentation and of the immobilized cell process are different.

Simulation investigations are proven to be powerful tools for evaluating the fermentation processes alternatives that degrease spending of expenses on pilot experiments. The quality of the simulation itself depends on the quality of the underlying mathematical model used foot prediction of the responses of a given system to changes in environmental and operating conditional. Hence the mathematical models should describe with sufficient accuracy the mechanisms of the processes under consideration. For the purpose of bioprocesses simulation, kinetic models based on mass balance of the main compounds in the bioreactor. Modeling batch fermentation process by the yeast Saccharomyces Carlsbergensis immobilized in Na-alginate gel beds were considered. They describe the main factors affecting the glucose concentration - substrate and product inhibition, but none of them can account simultaneously for all of these factors. However there is no model universal structure that could perfectly suit glucose fermentation by all possible kinds of strains since each particular strain has its specifics that require an individual approach to kinetics modeling.

The aim of our study was to carry out a comparative analysis of different mathematical structures known by far for modeling of batch alcoholic fermentation with free and immobilized cells of Saccharomyces Carlsbergensis using real experimental data. The two types of processes (with free and immobilized cells) were compared with respect to the main model parameters that determine the main interactions in the culture - inhibition and transformation of sugar to ethanol and biomass Conclusions were drawn about the influence of cell immobilization on the batch process. The main purpose of this study was to choose the best model that will be further refined and used for control synthesis of the process in order to increase its productivity. 


\section{MATERIALS AND METHODS}

The equipment consists in eight conical bioreactors (vessels) $250 \mathrm{ml}$, where was placed the immobilized yeast with the entrapment method and free yeast, in respective amount 1 and $2 \mathrm{~g} / \mathrm{l}$. For yeast cell immobilization solution of $\mathrm{Na}$ - alginate was used. It was prepared by dissolving alginate in distilled water at constraint stirring until a homogeneous solution was obtained. The immobilization has been carried out by cells inclusion into the alginate matrix, respecting the entrapment method. The following diameters of the biocatalyst spherical particles have been obtained: 4, 5.3 and $7 \mathrm{~mm}$. The fermentations have been carried out until a constant amount of glucose was taken, at the ambient temperature.

On average, every four hours was carried out measurements for determining the amount of glucose consumed and the amount of yeast which released for each samples. Measurements were carried out through the refractometer and the spectrophotometer (in $550 \mathrm{~nm}$ ).

\subsection{Mathematical models}

We chose simplified mass balance mathematical models that reflect only the kinetic rates of the main process reactions: biomass growth, ethanol production and substrate consumption for biomass and product formation.

The fermentation process kinetics was described with the ordinary differential equation:

$$
\begin{aligned}
& \frac{d X}{d t}=\mu X \\
& \frac{d P}{d t}=q X \\
& \frac{d S}{d t}=-\frac{1}{Y_{X / S}} \frac{d X}{d t}=-\frac{1}{Y_{P / S}} \frac{d P}{d t}
\end{aligned}
$$

Where $\mathrm{X}$ was biomass concentration, $\mathrm{P}$ is ethanol concentration, $\mathrm{S}$ substrate concentration, $\mathrm{Y}_{\mathrm{X} / \mathrm{S}}$ and $\mathrm{Y}_{\mathrm{P} / \mathrm{S}}$ were yield coefficients, $\mu$ and $q$ were specific growth and product accumulation rates.

The main kinetic parameters are:

- Maximal specifc growth rate $\mu_{\max }$,

- Monod constant, $\mathrm{K}_{\mathrm{s}}$ value is the concentration of substrate when $\mu$ is equivalent to half of $\mu_{\max }$

- The inhibitor constant, $\mathrm{K}_{\mathrm{i}}$ is an indication of how potent an inhibitor is, it is the concentration required to produce half maximum inhibition.

In these researches are used these models:

- $\quad$ Monod

$$
\mu=\mu_{\max } \frac{s}{s+K_{s}}
$$

- Contois

$$
\mu=\mu_{\max } \frac{s}{K_{s}^{*} X+s}
$$

- $\quad$ Teisser

$$
\mu=\mu_{\max }\left(1-\exp \left(-\frac{s}{K_{s}}\right)\right)
$$

- Substrate inhibition 


$$
\mu=\mu_{\max } \frac{s}{s+K_{s}} \exp \left(-\frac{s}{K_{i}}\right)
$$

- $\quad$ Product inhibition (Aiba)

$$
\mu=\mu_{\max } \frac{s}{s+K_{s}} \exp \left(-K_{i}^{*} p\right)
$$

Three linearization methods were used to determine $\mu_{\max }$ and Ks, which are:

- $\quad$ Lineweaver - Burk

$$
\frac{1}{\mu}=\frac{K_{s}}{\mu_{\max }} \frac{1}{s}+\frac{1}{\mu_{\max }}
$$

- Hans Woolf

$$
\frac{s}{\mu}=\frac{1}{\mu_{\max }} s+\frac{K_{s}}{\mu_{\max }}
$$

- $\quad$ Eadie Hofslee

$$
\mu=-K_{s} \frac{\mu}{s}+\mu_{\max }
$$

And the one with the highest correlation coefficient was chosen, and $\mathrm{K}_{\mathrm{i}}$ was determined by the mathematical method trial and error.

\section{RESULTS AND DISCUSSION}

The system differential equations (1) are a classic description of various biotechnologinal processes. We tested 12 mathematical models obtained by different authors and applied for different biotechnological processes. The careful analysis of that work and our experimental results restricted our choice to 5 of these 12 mathematical models. For some of the discarded models unusual and unexplained constant values, even from a biological point of view, were obtained, while in other models it was impossible to identify the parameters. The mathematical models chosen as a result of the pre-selection are listed: equation (2a) - (2e). The obtained parameters kinetic for all the investigated models are given in Table 1.

TABLE 1

IDENTIFIED MODEL PARAMETERS

\begin{tabular}{|c|c|c|c|c|c|c|c|}
\hline & & $\boldsymbol{\mu m a x}$ & $\mathbf{K s}$ & $\mathbf{K i}$ & $\mathbf{Y x} / \mathbf{s}$ & $\mathbf{Y p} / \mathbf{s}$ & $\mathbf{R}$ \\
\hline & $\mathbf{I m m o b i l i z e d ~ c e l l ~}$ & $\mathbf{1 / o r e}$ & ${ }^{\circ} \mathbf{B r i x}$ & ${ }^{\circ} \mathbf{B r i x}$ & - & - & $\%$ \\
\hline & $4 \mathrm{~mm}$ & 0.593 & 12.01 & - & 0.0011 & 0.5 & 97.6 \\
\hline Fermention 1 & $7 \mathrm{~mm}$ & 0.385 & 10 & & 0.0023 & 0.5 & 66.3 \\
\hline & Free cell & 0.711 & 14.413 & & 0.0988 & 0.5 & 89.3 \\
\hline & $4 \mathrm{~mm}$ & 0.471 & 20.94 & 104.7 & 0.0117 & 0.5 & 93.8 \\
\hline Fermention2 & $7 \mathrm{~mm}$ & 0.406 & 22.94 & 114.7 & 0.0127 & 0.5 & 97.4 \\
\hline & Free cell & 0.384 & 24.894 & 124.47 & 0.0669 & 0.5 & 91.6 \\
\hline Fermention 3 & $4 \mathrm{~mm}$ & 0.321 & 22.794 & 113.965 & 0.010099 & 0.5 & 95.17 \\
\hline & $7 \mathrm{~mm}$ & 0.412 & 27.629 & 138.145 & 0.013409 & 0.5 & 87.58 \\
\hline & Free cell & 0.208 & 23.637 & 118.19 & 0.062401 & 0.5 & 95 \\
\hline Fermention 4 & $4 \mathrm{~mm}$ & 0.135 & 14.79 & 0.1 & 0.0143 & 0.5 & 98.3 \\
\hline & $7 \mathrm{~mm}$ & 0.167 & 0.613 & 0.006 & 0.0217 & 0.5 & 80.7 \\
\hline & Free cell & 0.11 & 18.84 & 0.18 & 0.1148 & 0.5 & 97.1 \\
\hline Fermention 5 & $4 \mathrm{~mm}$ & 0.009 & 12.37 & 0.12 & 0.0059 & 0.5 & 99.9 \\
\hline & $7 \mathrm{~mm}$ & 0.011 & 12.51 & 0.12 & 0.0064 & 0.5 & 99.6 \\
\hline & Free cell & 0.00008 & 12.3 & 0.12 & 0.1975 & 0.5 & 99 \\
\hline
\end{tabular}


Figure 1. shows the model simulation result comparison with experimental data for the specific growth rate modeling equation. As seen, all the identified models describe with relatively good accuracy the batch fermentation process in the both cases (with and without cell immobilization). In order to choose the best model, it is important to consider how well it fit experimental data with Monod model.

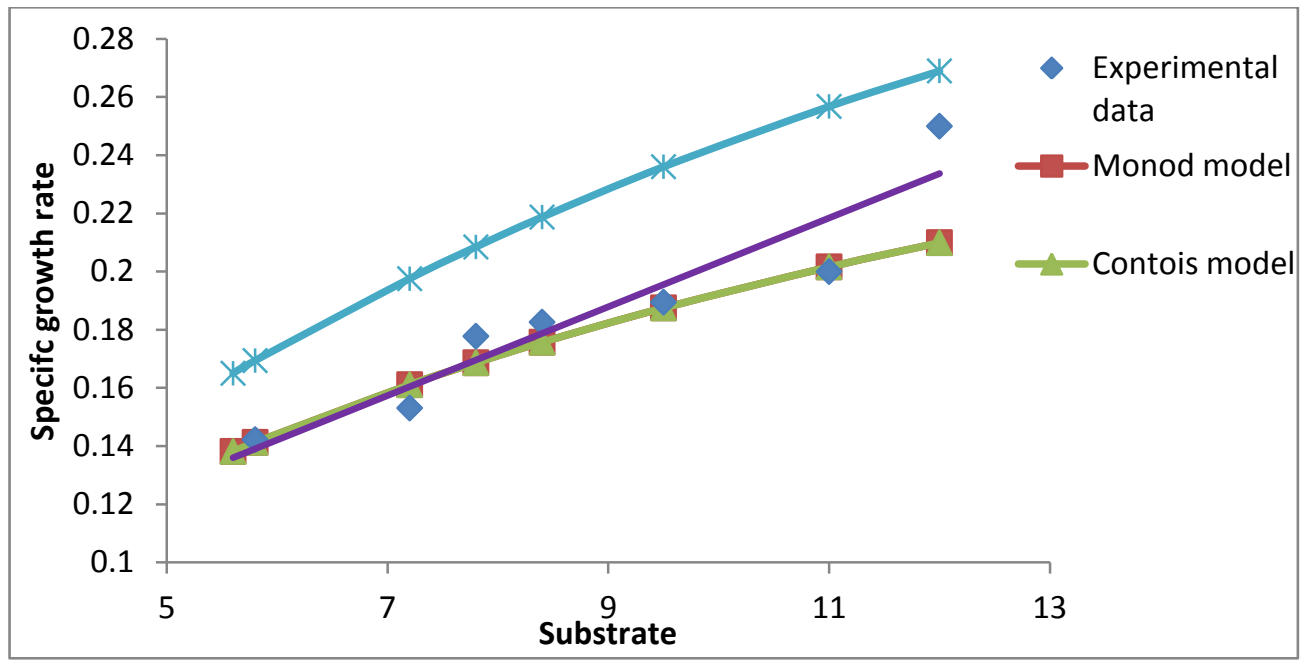

(A)

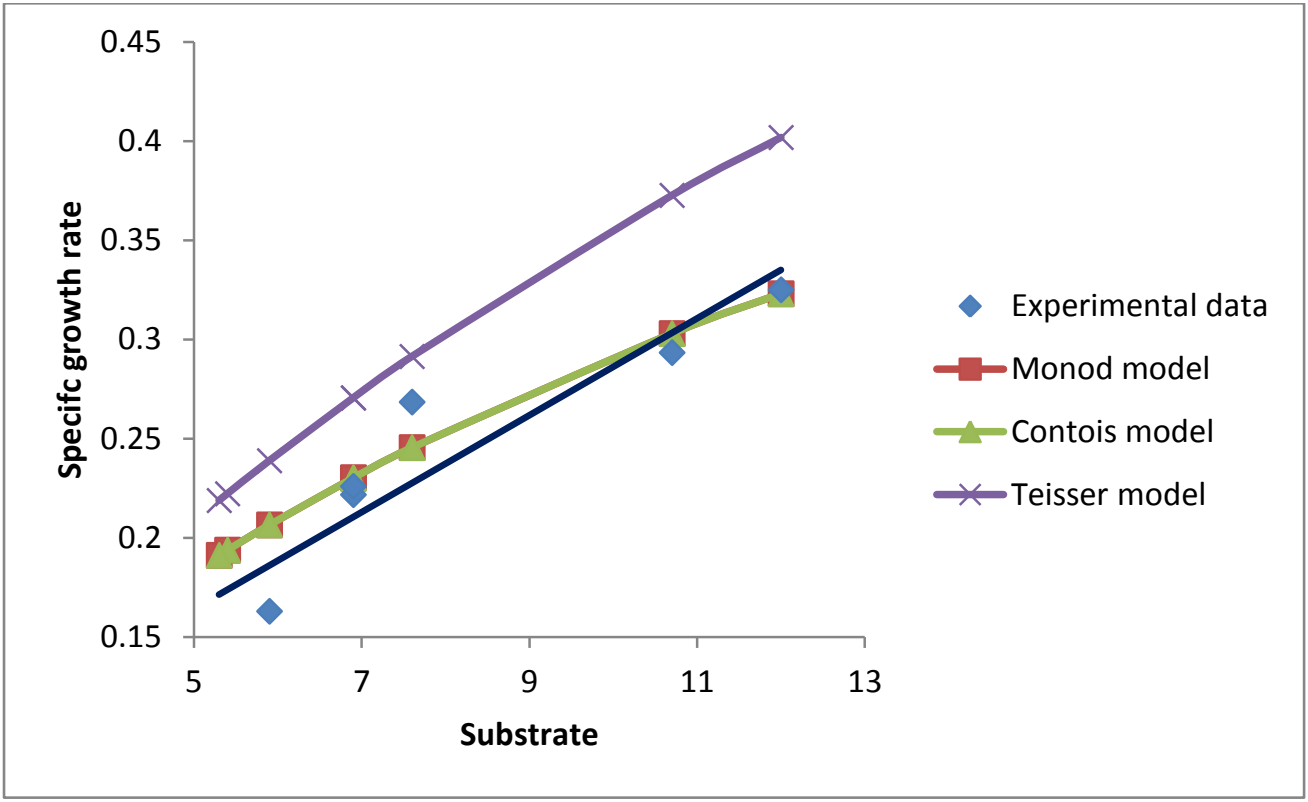

(B)

FIG. 1. COMPARISON OF MATHEMATICAL MODELS FOR SPECIFIC GROWTH RATE IN THE CASES OF IMMOBILIZED CELL (4 mm) (A) AND FREE CELLS (B), IN THE FIRST FERMENTATION.

Looking at Fig. (1b) we can conclude that in the case of free cell fermentation the model of Teisser do not fit in comparison to the rest of the rest of the models. In the case of the free cell fermentation, we have high values of the parameters $\mathrm{K}_{\mathrm{s}}$ and $\mathrm{Y}_{\mathrm{X} / \mathrm{S}}$. In low cell density Monod and Contois model fit well with experimental data, whereas in high cell density experimental data do not fit with any models.

A similar analysis can be done for the models of the second case of immobilized cell fermentation, Fig. 1a. Since immobilized cells growth inside beads pearls, they are restricted by diffusion resistances and have a lower maximum specific growth rate. The decreasing of the specific growth rate is due to the internal diffusion resistances rather than due to the external ones because the process is carried out with intensive stirring and hence the main resistance is due to the substrate diffusion through the alginate pores. In that case the models have lower value of $\mathrm{K}_{\mathrm{s}}$ in comparison with those for the free cell fermentation. This could be explained with higher concentration of viable yeasts cells in the bioreactor working volume. 
In Fig. 2. are given comparison of the mathematical models, in case of substrate inhibition.

Fig. (2a) shows cell immobilized (4mm) and we see that Monod, Contois and Teisser models do not fit at all with experimental data. From the results obtained fit the equation (2d), which has an exponential dependence. In this case we have the impact of the inhibition constant $\mathrm{K}_{\mathrm{i}}$, that reduces the maximum specific growth rate and increases the semi saturation constant $\mathrm{K}_{\mathrm{s}}$. In the Fig. (2b) performance is shown for the free cell fermentation and it also has an exponential dependence, due to the substrate inhibition maximum specific growth rate decrease more and the semi saturation constant $\mathrm{K}_{\mathrm{s}}$ and inhibition constant $\mathrm{K}_{\mathrm{i}}$ increase even more.

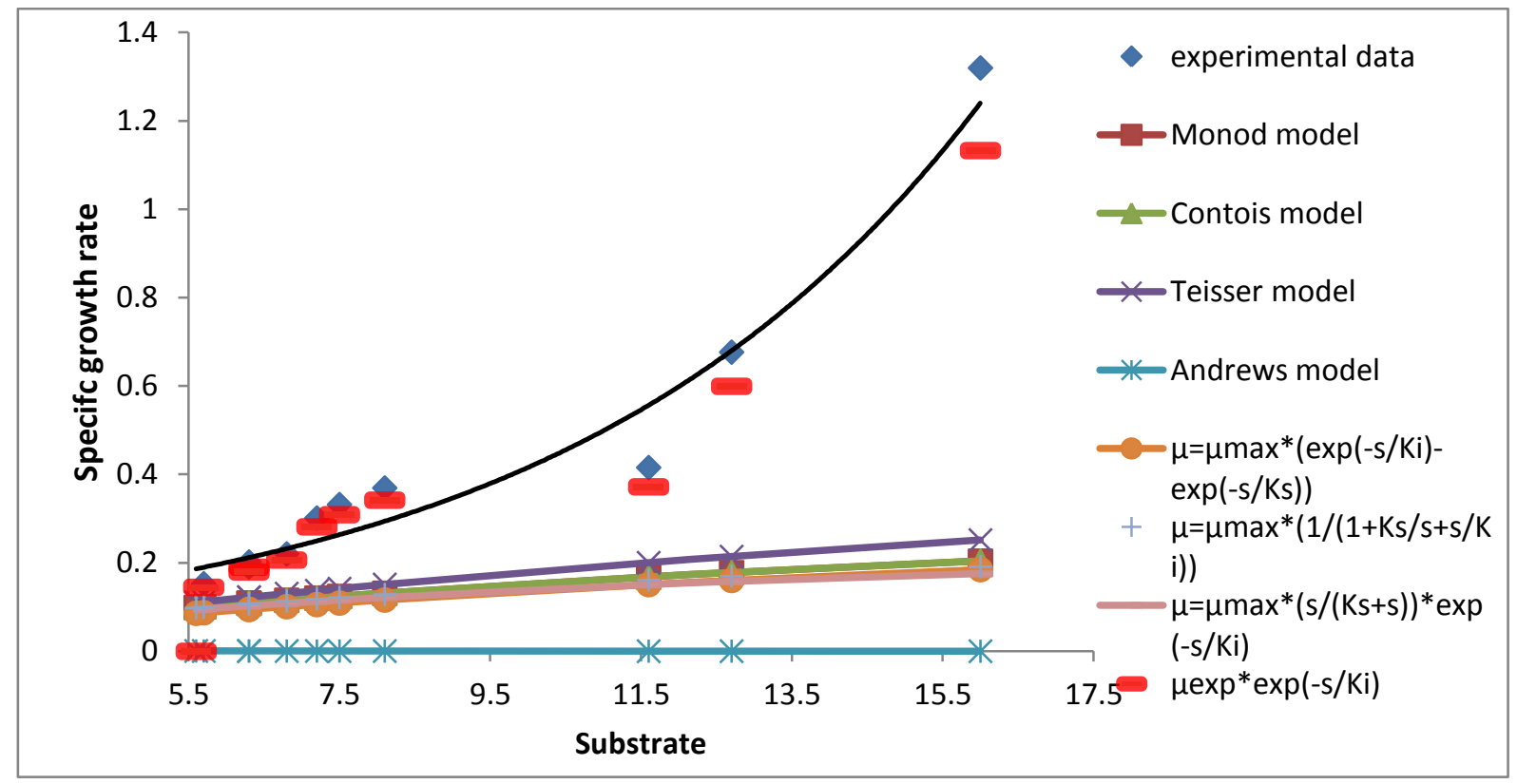

(A)

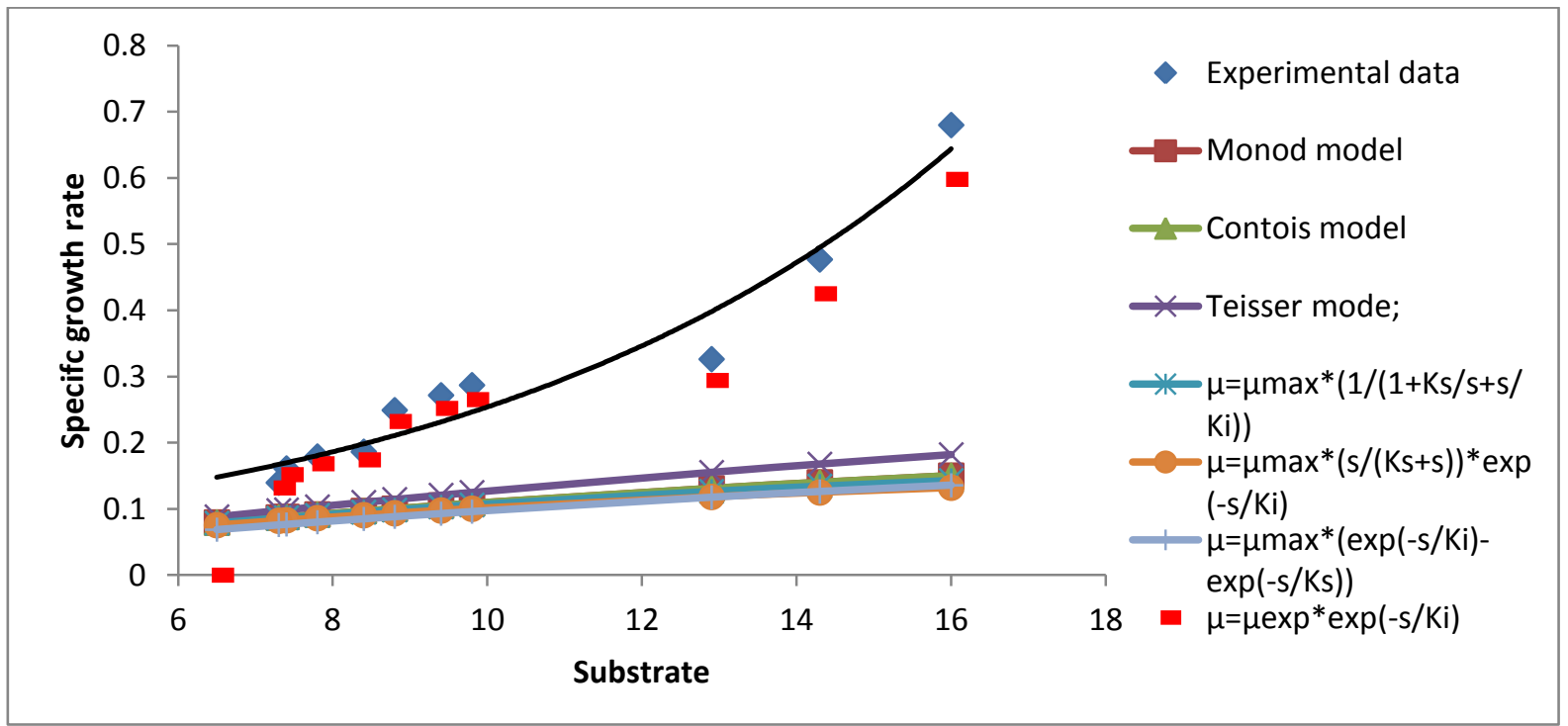

(B)

\section{FIG. 2. COMPARISON OF MATHEMATICAL MODELS FOR SPECIFIC GROWTH RATE IN THE CASES OF IMMOBILIZED CELL (4 mm) (A) AND FREE CELLS (B), IN THE SECOND FERMENTATION.}

In Fig. 3. are given comparison of the mathematical models, in case of product inhibition. Fig. (3a) shows cell immobilized $(4 \mathrm{~mm})$ and Monod, Contois and Teisser models are far from experimental data during the exponential cell growth. From the results obtained fit the equation (2e), which has an exponential dependence. The values of $\mathrm{K}_{1}$ parameter are very low. This is because they obtained ethanol concentration is much lower than the concentration that causes complete cell growth inhibition for our experimental data. In the Fig. (3b) performance is shown for the free cell fermentation and it also has an exponential dependence. 


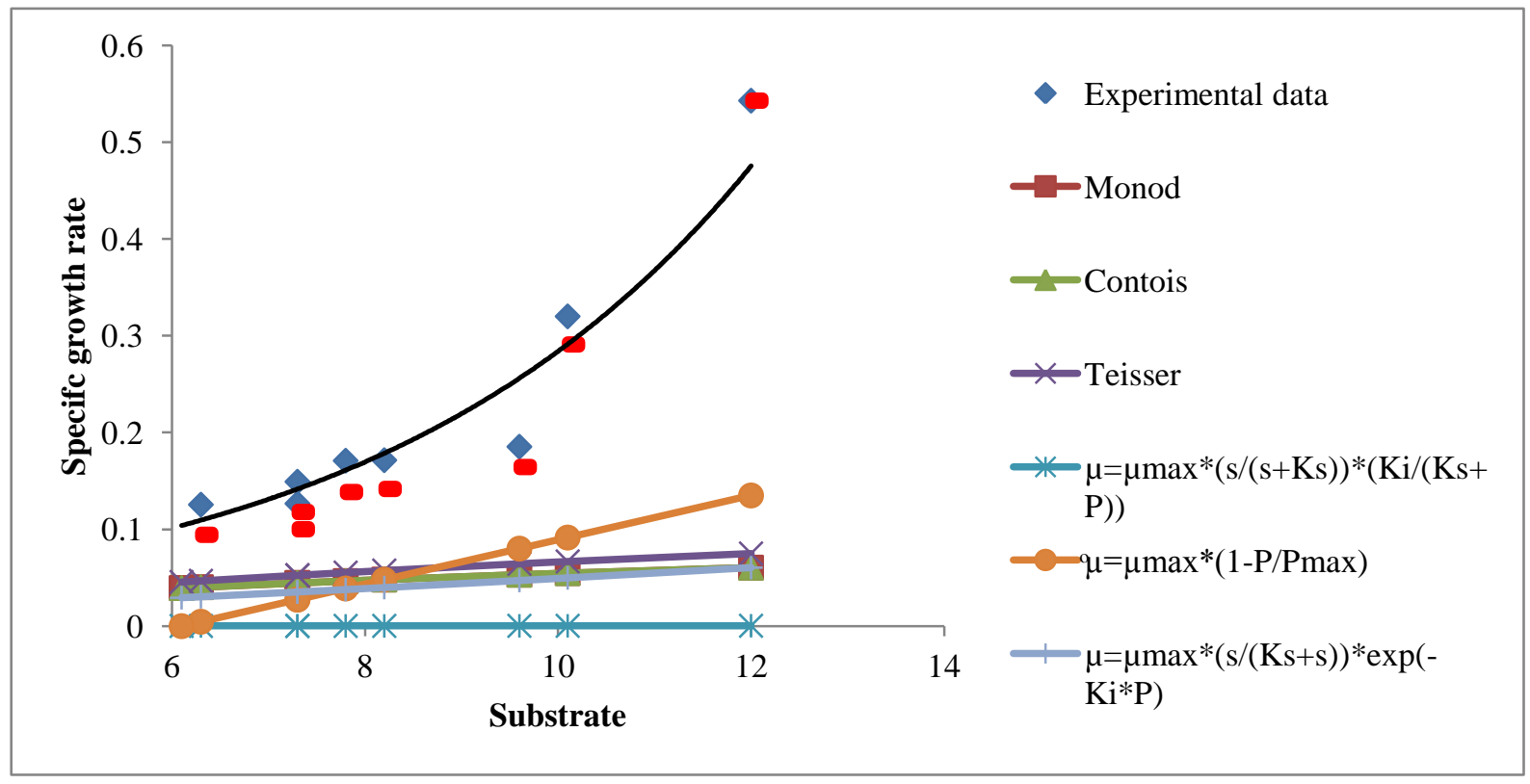

(A)

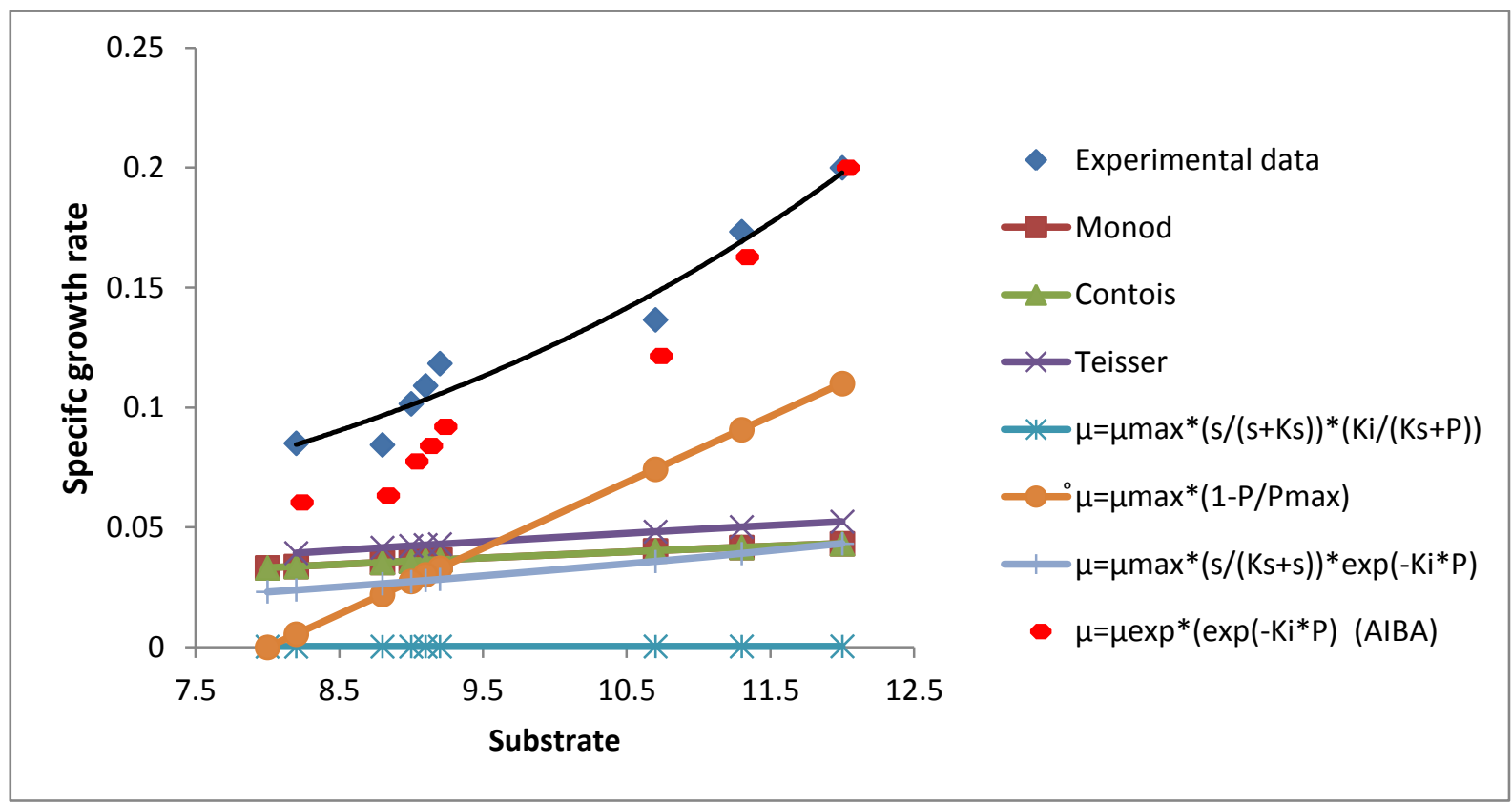

(B)

FIG. 3. COMPARISON OF MATHEMATICAL MODELS FOR SPECIFIC GROWTH RATE IN THE CASES OF IMMOBILIZED CELL (4 mm) (A) AND FREE CELLS (B), IN THE FOURTH FERMENTATION.

In the case of the fourth fermentation, where we have the product inhibition, see an approximation of the values of the kinetic parameters. Since the alginate beads have relatively big pores $(4,7 \mathrm{~mm})$, the diffusion through them is easy, i.e. the immobilizes grow in conditions similar to those of free fermentation. This can also explain the similar values of the maximum growth rate parameters $\mu_{\max }$ in both cases.

From the obtained results it is easy to choose a single best fitting model. Good approximation potential was shown by the models of Monod, Teisser, Aiba. The aim of this work was to make an selection of mathematical relationships, which begin the description of both the batch fermentation process with immobilized cells in the presence of diffusion resistances and continuous fermentation with immobilized cells. These relationships described the ethanol fermentation process very well and gave a clear idea of the process parameters influence on the kinetic characteristics. These models have the best approximating ability to describe the alcoholic fermentation with Saccharomyces Carlsbergensis. 


\section{CONCLUSION}

In the present investigation we considered modeling of batch fermentations with free and immobilized yeasts Saccharomyces Carlsbergensis in $\mathrm{Na}$ - alginate. Eleven of the most popular cultivation model structures were investigated. From the obtained results it is difficult to choose a single best fitting model. Good approximation potential was shown by the model of Monod, Teisser, Aiba. For substrate and product inhibition the most approximate models were exponential. Non-inhibitory fermentations fits with the Monod model, while product and substrate inhibition fermentation fits with exponential models.

As a conclusion immobilized cells in normal conditions ferment similarly to the free yeast. Based on the physical meaning of the obtained model parameters we compared the free and the immobilized cell fermentations. The results definitely proved the advantage of cell immobilization leading to higher production rates. Also immobilization based to kinetic parameters decrease the substrate and product inhibition phenomena compared to free yeast fermentation

\section{REFERENCES}

[1] Arellano-Plaza M, Herrera-López EJ, Díaz-Montaño DM, Moran A, Ramírez-Córdova JJ (2007). Unstructured Kinetic Model for Tequila Batch Fermentation. Int. J. Math Comput. Simul, Vol. 1, Num. 1.

[2] Bailey JE, Ollis DF (1986). Biochemical Engineering Fundamentals, Second Edition. USA: McGraw-Hill Book Company.

[3] Aiba S, Shoda M, Nagatani M. Kinetic of product inhibition in alcohol fermentation. Biotechnol Bioeng 2000; 67;671-90.

[4] Luljeta Xhagolli, Ilirjan Malollari. Inxhinieria e proceseve biokimike. 2009. 